\title{
ARTROPODOFAUNA DE SOLO ASSOCIADA À SERAPILHEIRA AO LONGO DE UM GRADIENTE TOPOGRÁFICO NA CHAPADA DOS GUIMARÃES, MATO GROSSO, BRASIL
}

\author{
M. R. MELLO', E. V. MASSONI JÚNIOR ${ }^{2}$, M. S. ALVES ${ }^{3}$ \\ ${ }^{1,2,3}$ Centro Universitário de Várzea Grande (UNIVAG) \\ michelly.rosa1@gmail.com ${ }^{1}$
}

Submetido 19/03/2016 - Aceito 30/04/2018

DOI: $10.15628 /$ holos. 2018.4300

\section{RESUMO}

$\mathrm{Na}$ serapilheira, a comunidade de artrópodes depende de fatores como o tipo de cobertura vegetal, o tipo de solo, o clima local e a diversidade de micro habitats. Esses fatores podem ser influenciados por diferentes variáveis ambientais. Sabendo-se que a serapilheira é o principal nutriente para a sobrevivência dos artrópodes e que a redução desta afetaria diretamente a diversidade de indivíduos, o objetivo deste trabalho foi inventariar a fauna de artrópodes relacionados à serapilheira ao longo do gradiente topográfico, analisando a influência das variáveis ambientais, na riqueza e abundância de indivíduos. As coletas foram realizadas na propriedade Chapada Aventura, Chapada dos Guimarães - MT, utilizando armadilhas de queda (pitfall). Posteriormente os espécimes coletados foram encaminhados ao Laboratório de Zoologia do UNIVAG Centro Universitário, Várzea Grande - MT, para a realização da triagem. O programa BioEstatc 5.0 foi utilizado para a análise da influência das variáveis ambientais sobre a comunidade. A ordem de maior representatividade foi Hymenoptera, totalizando $77,2 \%$ dos indivíduos coletados. Das variáveis analisadas, apenas o gradiente topográfico apresentou influência marginalmente significativa sobre a comunidade de artrópodes. Possivelmente, um estudo mais aprofundado com análise de mais variáveis possa apresentar resultados mais relevantes para a consolidação das hipóteses levantadas nesta pesquisa.

PALAVRAS-CHAVE: Artrópodes, serapilheira, gradiente topográfico.

\section{SOIL ARTHROPOD ASSOCIATED BURLAP ALONG A GRADIENT IN TOPOGRAPHICAL CHAPADA OF GUIMARAES , MATO GROSSO , BRAZIL}

\begin{abstract}
In the burlap arthropods community depends on factors like the type of vegetation cover, soil type, climate local and diversity of microhabitats. These factors can be influenced by different enveronmental variables. Knowing that the burlap is the main nutrient for survival fos arthropods and that this reduction would directly affect the diversidad of individuals, the objective of this work it was to inventory the fauna of arthropods related to litter along a topographic gradient, analyzing the influence of environmental variables, wealth and abundance of individuals. Samples were collected on the property Chapada Aventura, Chapada dos Guimarães-
\end{abstract}

MT, using pitfall traps. Latter the collected specimens were sent to the Zoology lab UNIVAG Centro Universitário, Várzea Grande- MT for the realization of the same screening. The program BioEstatc 5.0 was used for the analysis of the influence of environmental variables on the community. The order was the most representative Hymenoptera, totaling $77,2 \%$ individuals gathered. Of the variables analyzed, only the topographic gradient showed marginally significant influence on arthropod community. Possibly further study with analysis of more variables can display more relevant results for the hypotheses for this search.

KEYWORDS: Arthropods, burlap, topographic gradient. 


\section{INTRODUÇÃO}

Os sistemas tropicais possuem baixa fertilidade apresentando-se bastante intemperizados. Nesses sistemas, a deposição de matéria orgânica é considerada a maior fonte de nutrientes, sendo assim de vital importância para a manutenção da vida animal e vegetal (CORREIA et al., 2000).

A matéria orgânica proveniente da parte aérea das plantas como as folhas, os frutos e os galhos das árvores, que se deposita sobre o solo, é conhecida como serapilheira (CORREIA et al., 2000). Existem vários fatores que podem agir interferindo na deposição dessa matéria orgânica, dentre eles os que mais apresentam destaque são o clima, o solo e a formação vegetal (GONZALES et al., 1982).

A fauna de artrópodes associada à serrapilheira destaca-se pelo fato desses animais participarem de diferentes níveis tróficos (ZARDO et al., 2010), exercendo também variadas funções dentro do ecossistema, alterando suas propriedades físicas, químicas e biológicas, promovendo assim a fragmentação da matéria orgânica e a estruturação do solo (FERREIRA et al., 1998).

A artropodofauna apresenta uma tendência de acompanhar a riqueza vegetal, pois, muitas espécies, dependem direta ou indiretamente dos recursos disponibilizados por ela (BATTIROLA, 2005). O filo Arthropoda apresenta uma grande capacidade adaptativa, ocupando variados nichos e micro habitats. Por essa razão, ele tem sido considerado um dos grupos com maior sucesso na colonização de habitats terrestres (RUPPERT et al., 2005), sendo considerado um dos maiores representantes em abundânica de invertebrados de solo, desenvolvendo grande função ecológica na ciclagem de nutrientes e na manutenção da biodiversidade local. A comunidade de artrópodes de solo responde às mudanças causadas em seu habitat, podendo estas respostas, alterar o funcionamento de um ecossistema (RICHARDS, 1974). Em serapilheira, a comunidade de artrópodes depende de fatores como o tipo de cobertura vegetal, o tipo de solo, o clima local e a diversidade de micro habitats (MUDREK; MASSOLI, 2014), sendo que esses fatores podem ser influenciados direta e indiretamente por diferentes variáveis ambientais. Segundo Oliveira (2013), o gradiente topográfico é uma importante variável de análise na diversidade de espécies de formigas. Em seu trabalho o autor enfatizou que existe um menor acúmulo de serapilheira nas áreas de declive em relação às áreas planas.

A declividade do terreno e a redução da serrapilheira poderia afetar diretamente a diversidade de 14 indivíduos. Estudos que analisem a relação entre a fauna de artrópodes de serrapilheira e o gradiente topográfico podem contribuir para o entendimento da estrutura dessas comunidades, como para a utilização destes como bioindicadores da qualidade do solo.

Diante do proposto, no presente trabalho objetivou-se inventariar a fauna de artrópodes relacionados à serapilheira ao longo do gradiente topográfico, analisando a influência das variáveis ambientais, na riqueza e abundância de indivíduos.

\section{METODOLOGIA}

\section{1 Área de estudo}


Localizada cerca de $40 \mathrm{~km}$ ao nordeste do município de Cuiabá, no estado de Mato Grosso, a Chapada dos Guimarães possui uma das principais unidades de conservação de proteção integral do cerrado mato-grossense. Por esta razão a região apresenta uma extensa área de vegetação nativa (LOPES et al., 2008).

A paisagem local é composta por diferentes fitofisionomias de Cerrado, dentre elas as matas de galeria, as matas secas deciduais e semideciduais, e os campos rupestres que ocorrem em afloramentos rochosos, em altitudes acima de 800 metros (LOPES et al., 2008). O clima local é caracterizado por um inverno seco que se estende entre maio e setembro e um verão chuvoso que se estende entre outubro e abril (NIMER, 1979).

O local escolhido para o desenvolvimento das amostragens foi à propriedade Chapada Aventura (Figura 1), localizada no início do Vale da Benção, na cidade de Chapada dos Guimarães, MT. A propriedade apresenta uma vegetação típica de cerrado e abriga uma grande diversidade de espécies.
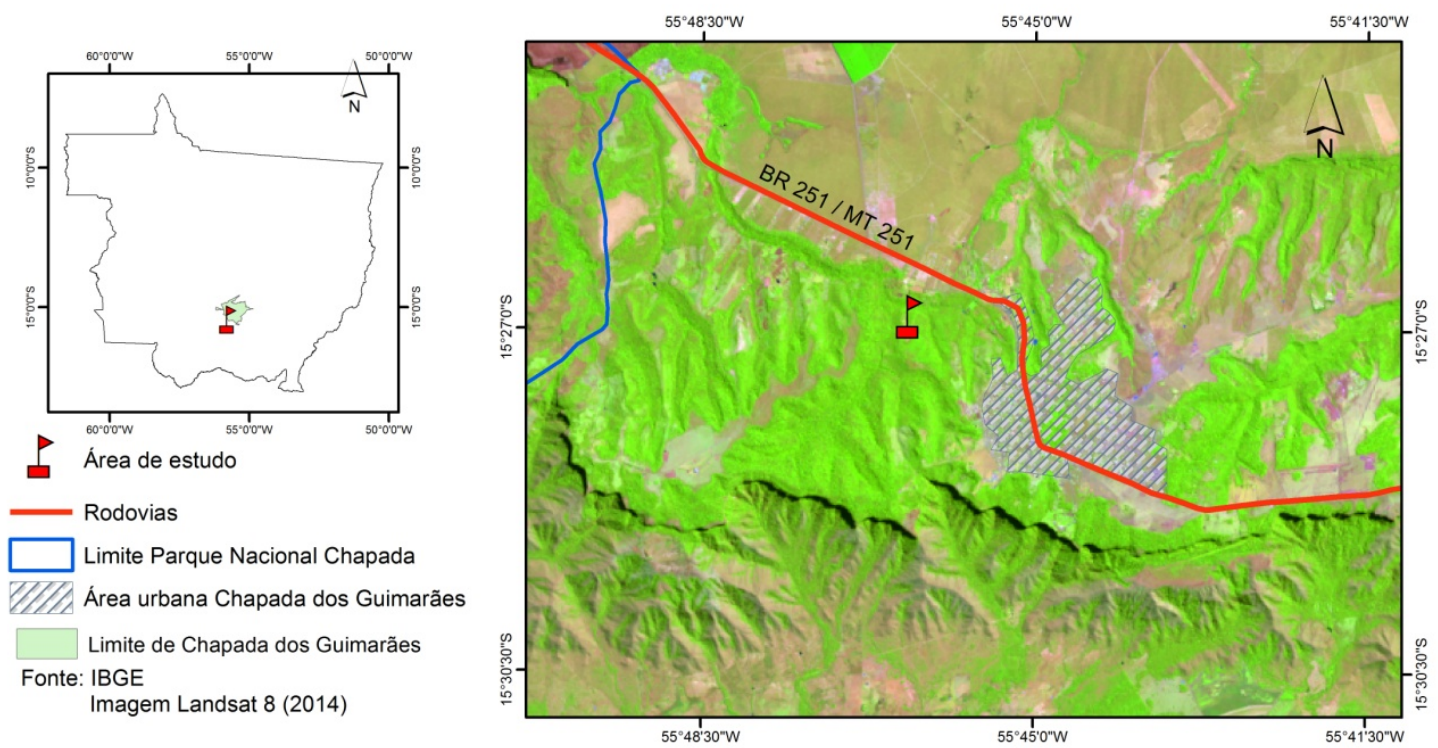

Figura 1: Propriedade Chapada Aventura, Chapada dos Guimarães, Mato Grosso, Brasil.

Fonte: IBGE, imagem Landsat 8, 2014.

As amostragens foram realizadas em uma área de declive pouco antropizada com extensão de 800 metros, localizada a aproximadamente 35 metros do córrego Cangas, em trecho com fitofisionomia predominante de mata de galeria.

\subsection{Amostragens}

As coletas foram realizadas nos dias 14 e 15 de julho de 2015, por meio de armadilhas de queda (pitfall), confeccionadas com garrafas pet com capacidade para 2 litros, cortadas na região superior com aproximadamente $30 \mathrm{~cm}$, contendo em seu interior $200 \mathrm{ml}$ de álcool $70 \%$ e duas gotas de detergente. 
Foram instaladas 30 armadilhas dispostas, ao longo do gradiente topográfico, distribuídas em 10 pontos, sendo que em cada ponto foram instaladas três armadilhas distante entre si em 4 metros, sendo duas réplicas. Para a obtenção da elevação de cada ponto foi utilizado um GPS Garmin Etrex Venture HC. A elevação inicial foi de 658 metros e a final de 624 metros.

A altura da serapilheira foi medida com o auxílio de uma fita métrica, em três etapas: na primeira foi feita padronização de um raio de 3 metros no entorno dos pontos, para que fosse realizada a contagem de árvores dentro de cada raio. Na segunda etapa, foi estabelecido o valor de altura da serapilheira de cada réplica, medido ao lado esquerdo de cada pitfall. Na terceira etapa, foi estabelecida a média de altura de serapilheira através da soma dos valores obtidos nas amostras de cada ponto.

As armadilhas ficaram abertas por um período de 24 horas, sendo que decorrido este período as mesmas foram retiradas e o material coletado foi acondicionado em potes plásticos, contendo $200 \mathrm{ml}$ de álcool 70\% lacrados e transportados até o laboratório de Zoologia, do UNIVAG Centro Universitário, Várzea Grande, MT.

No laboratório, o material coletado passou por uma limpeza com o auxílio de uma peneira, pinça e água, com o intuito de separar os artrópodes das partículas do solo. Posteriormente, as amostras de cada ponto foram somadas e, assim, foi realizada a contagem e identificação dos indivíduos coletados em uma resolução taxonômica até o nível de ordem. Após a obtenção das ordens, os indivíduos coletados foram separados em morfoespécies pelo método de morfoespeciação. A abundância de artrópodes foi obtida a partir do número total de indivíduos de cada ordem, coletados em cada ponto e a riqueza foi obtida a partir do número total de morfoespécies de cada ponto.

O programa BioEstatc 5.0 foi utilizado para a obtenção do valor de regressão linear simples de cada variável, para que assim fossem formulados gráficos de dispersão para a verificação do efeito do gradiente topográfico, altura da serapilheira e número de árvores sobre a abundância e a riqueza dos artrópodes coletados.

\section{RESULTADOS E DISCUSSÃO}

Foi observado que houve uma variação da altura da serapilheira dos pontos amostrais entre 2,3-6,3 cm. O volume e a deposição de serapilheira o solo pode ser influenciada pelo número de árvores, pela abertura do dossel (ZARDO et al., 2010), e, pela disposição do gradiente topográfico (OLIVEIRA, 2013).

Esperava-se encontrar um maior acúmulo de serapilheira nos pontos amostrais onde foi encontrado um maior número de árvores. Entretanto, pontos amostrais com poucas árvores também apresentaram altura de serapilheira elevada.

O gradiente topográfico analisado variou entre 658 - 624 metros. Toledo et al. (2011), enfatizam que locais inclinados podem apresentar menor acúmulo de serapilheira e maior espaçamento entre árvores. Possivelmente, a declividade da área de estudo com variação do gradiente de 34 metros, pode estar promovendo a movimentação da serrapilheira de um ponto para outro, afetando o acúmulo desta serapilheira nos pontos amostrais. Os resultados obtidos nas variáveis ambientais analisadas estão dispostos na tabela 1. 
Tabela 1 - Variáveis e atributos da comunidade analisados neste trabalho.

\begin{tabular}{ccccc}
\hline \multicolumn{2}{c}{ Variáveis ambientais } & \multicolumn{3}{c}{ Atributos da comunidade } \\
\hline $\begin{array}{c}\text { Altura da } \\
\text { Serapilheira } \\
(\mathbf{c m})\end{array}$ & $\begin{array}{c}\text { Altitude } \\
(\mathbf{m})\end{array}$ & $\begin{array}{c}\mathbf{N}^{\circ} \\
\text { Árvores }\end{array}$ & $\begin{array}{c}\text { Abundância } \\
\text { Riqueza de } \\
\text { morfoespécies }\end{array}$ \\
\hline 4,1 & 658 & 11 & 20 & 7 \\
4,3 & 656 & 15 & 61 & 5 \\
5,3 & 654 & 18 & 68 & 5 \\
6 & 649 & 15 & 14 & 8 \\
3 & 641 & 7 & 17 & 8 \\
4 & 638 & 8 & 85 & 12 \\
4,6 & 635 & 9 & 14 & 7 \\
2,3 & 630 & 11 & 27 & 9 \\
5,6 & 627 & 7 & 14 & 8 \\
6,3 & 624 & 11 & 10 & 5 \\
\hline
\end{tabular}

Foi coletado um total de 330 artrópodes de solo, distribuídos em três classes: Insecta $(92,6 \%)$, Arachinida (6,9\%) e Chilopoda (0,3\%). As ordens com maiores abundâncias foram a Hymenoptera com 77,2\% ( $n=255$ ) subdivididos em 6 morfoespécies, seguida da ordem Araneae com 6,9\% ( $n=23$ ) subdividida em 12 morfoespécies e a ordem Coleoptera com 6,4\% ( $n=21$ ) subdividida em 4 morfoespécies. As outras ordens coletadas representaram 9,5\% ( $n=31)$, do total observado. A abundância relativa de espécimes coletadas em cada ordem esta disposta na tabela 2.

Tabela 2 - Abundância relativa e número de morfoespécies obtidos em cada ordem.

\begin{tabular}{lccc}
\hline \multicolumn{1}{c}{ Classe/Ordem } & $\begin{array}{c}\text { No de } \\
\text { indivíduos } \\
\text { coletados }\end{array}$ & $\begin{array}{c}\mathbf{N}^{\circ} \text { de } \\
\text { morfoespécies } \\
\text { coletadas }\end{array}$ & $\begin{array}{c}\text { \% de espécimes } \\
\text { Coletadas }\end{array}$ \\
\hline Insecta/Hymenoptera & 255 & 6 & 77,2 \\
Insecta/Coleoptera & 21 & 4 & 6,4 \\
Insecta/Hemiptera & 6 & 2 & 1,8 \\
Insecta/Orthoptera & 15 & 2 & 4,5 \\
Chilopoda/Scolopendromorpha & 1 & 1 & 0,3 \\
Insecta/Blattodea & 9 & 3 & 2,7 \\
Arachinida/Araneae & 23 & 5 & 6,9 \\
\hline Total & 330 & 23 & 100 \\
\hline
\end{tabular}

A ordem Hymenoptera foi à ordem de maior abundância com $77,2 \%(n=255)$, sendo também a ordem de maior riqueza com um total de 6 morfoespécies coletadas. Resultados semelhantes foram obtidos em estudos realizados por Mudrek; Massoli (2014), Zardo et al. (2010) e Rocha et al. (2010), onde os autores, analisando a comunidade de artrópodes em serrapilheira, também registraram a ordem Hymenoptera como uma das ordens de maior abundância em cerrado. Esta maior representatividade da ordem Hymenoptera também foi 
observada em fragmentos de mata na floresta amazônica (SILVA et al., 2011),e na caatinga (SANTOS et al., 2012). Já se previa este resultado, uma vez que, a ordem participa de grande parte dos processos ecológicos (BATTIROLA et al., 2005), fragmentando a serapilheira, retirando dela resíduos ricos em nutrientes e concentrando-os no solo, tornando-o assim mais rico em matéria orgânica (WAGNER et al., 2006).

A ordem com menor abundância e menor riqueza foi a Scolopendromorpha sendo coletado apenas um indivíduo no ponto 4 , representando $0,3 \%$ do total coletado. 0 ponto quatro apresentou uma altura de serrapilheira de $6 \mathrm{~cm}$ e um total de 15 árvores em seu entorno. Essas duas variáveis em conjunto podem estar influenciando a existência deste táxon neste ponto amostral, tendo em vista que, eles preferem ambientes escuros e úmidos (KNYSAK; MARTINS, 1999), e se escondem na serrapilheira depositada sobre o solo (COUTO, 2008). Os indivíduos desta ordem também são menos ativos, o que dificulta as chances de coleta.

O ponto de maior representatividade de indivíduos coletados foi o ponto 6 . Provavelmente, a serrapilheira neste ponto amostral, encontra-se ali depositada por um longo período de tempo, o que aumentaria o seu grau de decomposição. A serrapilheira com alto grau de decomposição apresenta uma grande quantidade de nutrientes, isso também aumenta a umidade relativa da camada superficial do solo e diminui a temperatura máxima e as oscilações de temperatura deste solo durante o dia. Estes nutrientes atraem os artrópodes para este ponto amostral, pois, eles atuam na ciclagem destes nutrientes através da fragmentação da serrapilheira.

Em contraposição ao esperado, o ponto 10 foi o ponto com menor representatividade de indivíduos coletados. Por apresentar um maior acúmulo de serapilheira, esperava-se encontrar neste ponto uma maior abundância e consequentemente uma maior riqueza de indivíduos. Possivelmente, a abundância e a riqueza de indivíduos neste ponto amostral podem estar sendo influenciadas pelo menor estágio de decomposição da serrapilheira, ou seja, um acúmulo mais recente desta matéria orgânica. A distribuição espacial das ordens e suas respectivas morfoespécies estão dispostos na tabela 3. 
Tabela 3 - Distribuição das ordens e morfoespécies nos pontos amostrais.

\begin{tabular}{|c|c|c|c|c|c|c|c|c|c|c|c|}
\hline & & P1 & $\mathbf{P 2}$ & P3 & P4 & P5 & P6 & P7 & P8 & P9 & P10 \\
\hline \multirow[t]{7}{*}{ Ordem } & Hymenoptera & & & & & & & & & & \\
\hline & Hymenoptera sp.1 & $x$ & $x$ & $x$ & $x$ & $x$ & $x$ & & & $x$ & $x$ \\
\hline & Hymenoptera sp.2 & & $x$ & & & $x$ & $x$ & & $x$ & $x$ & $x$ \\
\hline & Hymenoptera sp.3 & & $x$ & $x$ & & & & & & & \\
\hline & Hymenoptera sp.4 & & & & $x$ & & $x$ & & $x$ & & \\
\hline & Hymenoptera sp.5 & & & & $x$ & & & & $x$ & & \\
\hline & Hymenoptera sp.6 & & & & & & $x$ & $x$ & & & \\
\hline \multirow[t]{5}{*}{ Ordem } & Coleoptera & & & & & & & & & & \\
\hline & Coleoptera sp.1 & $x$ & & $x$ & $x$ & $x$ & $x$ & & $x$ & & \\
\hline & Coleoptera sp. 2 & $x$ & & & & & $x$ & $x$ & $x$ & $x$ & \\
\hline & Coleoptera sp.3 & & & & $x$ & $x$ & & & $x$ & & \\
\hline & Coleoptera sp.4 & & & & & $x$ & & & & & $x$ \\
\hline \multirow[t]{3}{*}{ Ordem } & Hemiptera & & & & & & & & & & \\
\hline & Hemiptera sp.1 & & $x$ & & & & & $x$ & & & \\
\hline & Hemiptera sp.2 & & & & & & & & & $x$ & \\
\hline \multirow[t]{3}{*}{ Ordem } & Orthoptera & & & & & & & & & & \\
\hline & Orthoptera sp.1 & $x$ & & $x$ & & $x$ & $x$ & & & $x$ & \\
\hline & Orthoptera sp.2 & & & & & & & $x$ & & $x$ & \\
\hline \multirow[t]{2}{*}{ Ordem } & Scolopendromorpha & & & & & & & & & & \\
\hline & Scolopendromorpha sp.1 & & & & $x$ & & & & & & \\
\hline \multirow[t]{4}{*}{ Ordem } & Blattodea & & & & & & & & & & \\
\hline & Blattodea sp. 1 & & & $x$ & $x$ & $x$ & $x$ & & & & \\
\hline & Blattodea sp. 2 & & & & & & $x$ & & & $x$ & \\
\hline & Blattodea sp. 3 & & & & & & & & $x$ & & \\
\hline \multirow[t]{8}{*}{ Ordem } & Araneae & & & & & & & & & & \\
\hline & Araneae sp. 1 & $x$ & $x$ & & $x$ & & $x$ & $x$ & & & \\
\hline & Araneae sp. 2 & $x$ & & & & $x$ & $x$ & $x$ & $x$ & & \\
\hline & Araneae sp. 3 & $x$ & & & & & & & $x$ & $x$ & $x$ \\
\hline & Araneae sp. 4 & & & & & & $x$ & $x$ & & & \\
\hline & Araneae sp. 5 & & & & & & & & & & $x$ \\
\hline & Abundância & 20 & 61 & 68 & 14 & 17 & 85 & 14 & 27 & 14 & 10 \\
\hline & Riqueza & 7 & 5 & 5 & 8 & 8 & 12 & 7 & 9 & 8 & 5 \\
\hline
\end{tabular}

As ordens de maior distribuição espacial foram Hymenoptera, distribuída em 100\% dos pontos amostrais, seguida de Coleoptera e Araneae, ambas distribuídas em $90 \%$ dos pontos amostrais. Foi obtido um total de 23 morfoespécies distribuídas pelos diferentes pontos amostrais. A distribuição das morfoespécies nos pontos amostrais está disposta na tabela 3. As morfoespécies de maior distribuição espacial foram Hymenoptera sp. 1, sendo encontrada em 8 dos 10 pontos amostrais, Hymenoptera sp. 2 e Coleoptera sp. 1, ambas coletadas em 6 dos 10 pontos amostrais.

A variável gradiente topográfico analisada neste trabalho, não apresentou influências significativas na riqueza $\left(r^{2}=0,073 ; p=0,450\right)$ de indivíduos da área coletada. Porém, foi observado influências marginalmente significativas na abundância $\left(r^{2}=0,383 ; p=0,056\right)$, dos indivíduos da mesma área (Figuras 2 e 3). Cardozo et al. (2009), analisando a influência da altitude sobre a comunidade de artrópodes do solo, levantaram a hipótese de que existe um 
aumento da biomassa da artropodofauna em gradientes de baixa amplitude, pois têm-se menor disponibilidade de nichos distintos, e à medida que a altitude eleva-se, estes poucos nichos apresentam-se mais especializados, agrupando artrópodes especialistas a esses nichos. Oliveira (2013), analisando a diversidade de formigas, sugeriu que, o número de espécies capaz de se estabelecer em áreas inclinadas é reduzido. Segundo o autor, locais inclinados podem apresentar menores taxas de substituição de espécies, apresentando assim, uma menor diversidade em relação ao gradiente topográfico.

Conforme enfatizam Luizão et al. (2004), áreas de declínio apresentam um menor acúmulo de serapilheira quando comparadas a áreas mais planas. A deposição de serrapilheira pode estar sendo influenciada pela variação do gradiente topográfico, o que possivelmente afeta a riqueza, abundância e distribuição espacial dos indivíduos coletados. Os resultados obtidos a partir da análise da influencia das variáveis ambientais sobre a abundância e riqueza da artropodofauna dos pontos amostrais, estão representados nas figura 2 e 3.
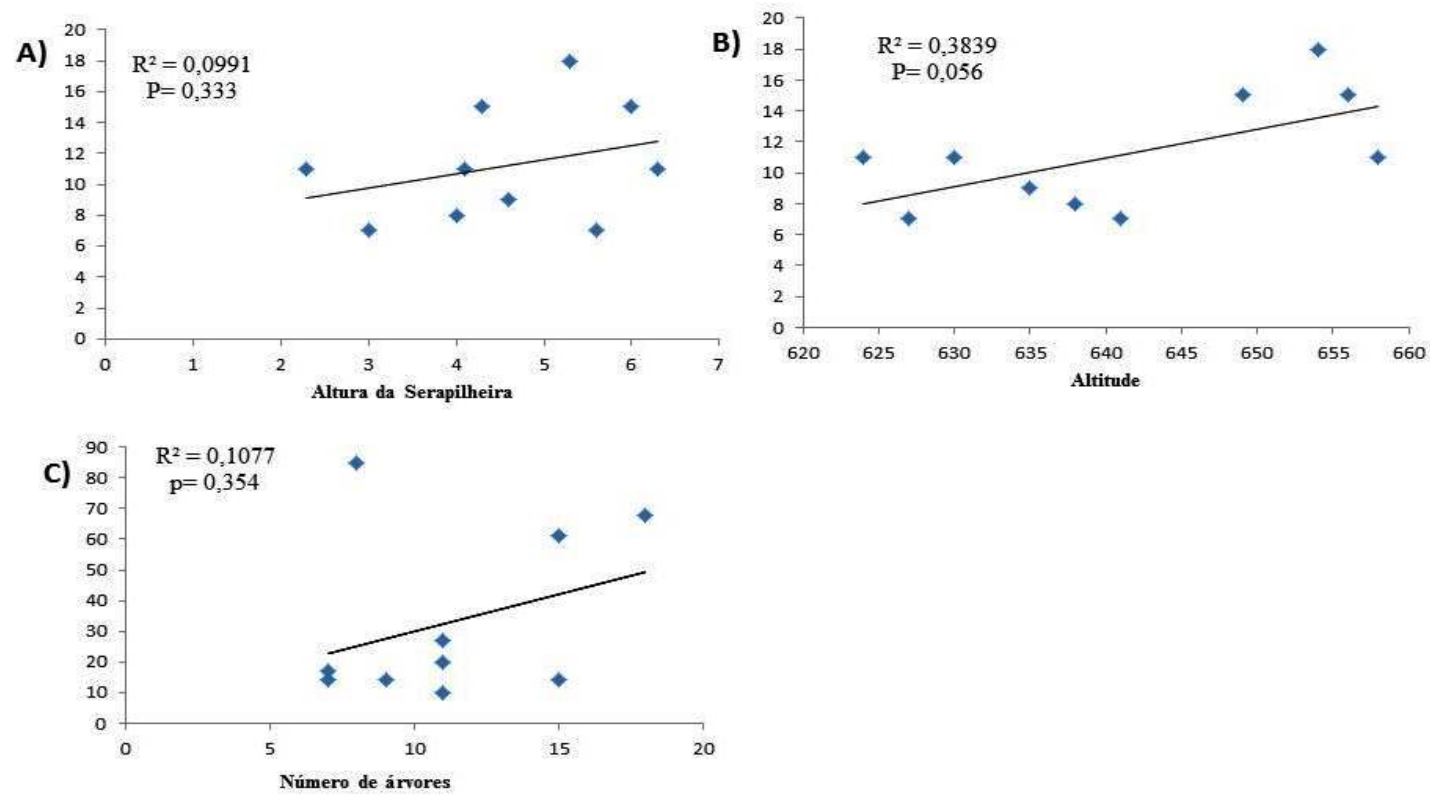

Figura 2: A) Análise de regressão linear simples da abundância de artrópodes em relação a altura da serapilheira

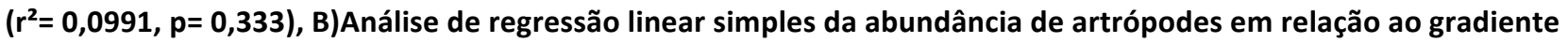
topográfico $\left.\left(r^{2}=0,3839, p=0,056\right), C\right)$ Análise de regressão linear simples da abundância de artrópodes em relação ao número de árvores $\left(r^{2}=0,1077, p=0,354\right)$. 

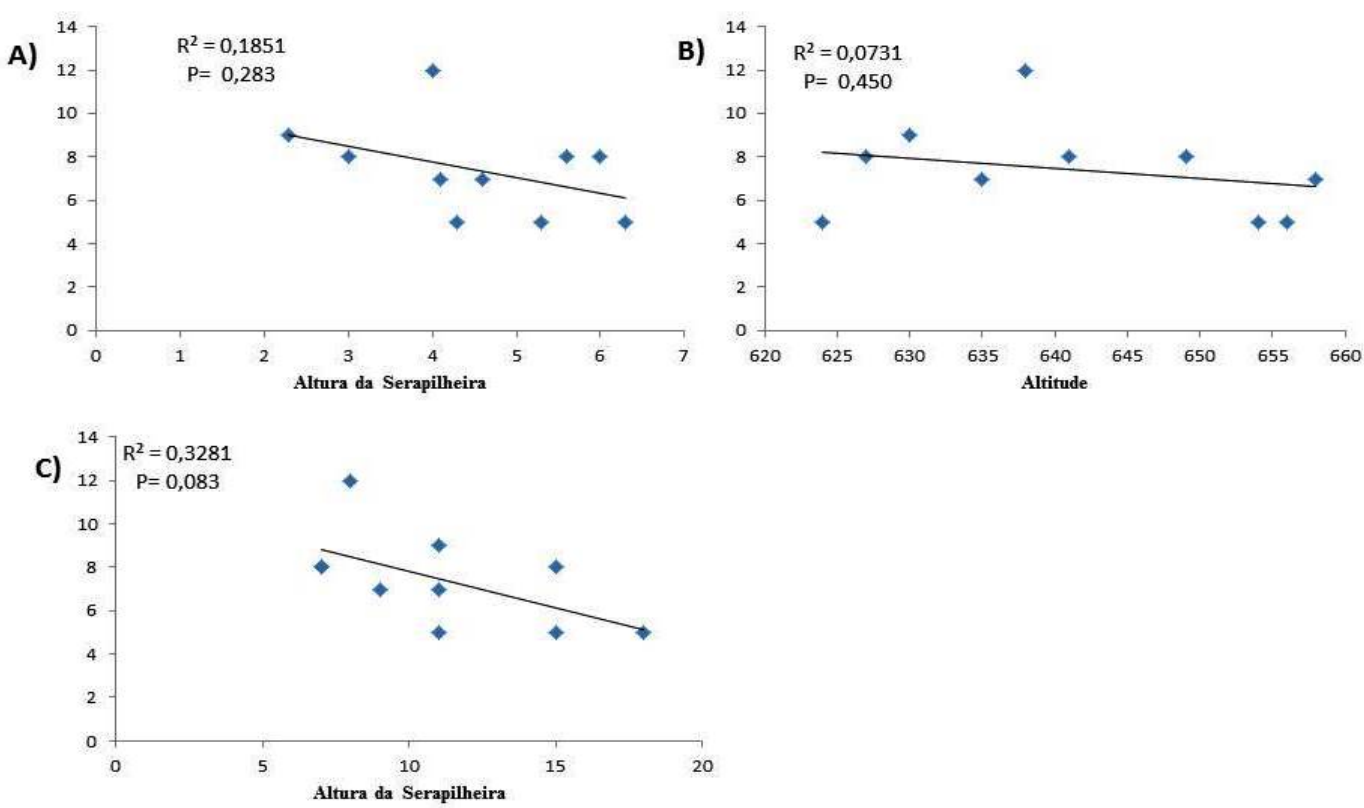

Figura 3 - A) Análise de regressão linear simples da riqueza de artrópodes em relação a altura da serapilheira $\left(r^{2}=\right.$ $0,1851, p=0,283), B)$ Análise de regressão linear simples da riqueza de artrópodes em relação ao gradiente topográfico $\left.\left(r^{2}=0,0731, p=0,450\right), C\right)$ Análise de regressão linear simples da riqueza de artrópodes em relação ao número de árvores $\left(r^{2}=0,3281, p=0,083\right)$.

Os resultados obtidos entre a riqueza de indivíduos e o gradiente topográfico, podem ser explicados pelo fato de que o gradiente topográfico analisado, em contraposição ao esperado, não foi suficiente para a obtenção de diferenças expressivas na artropodofauna de solo. Possivelmente gradientes topográficos com uma maior amplitude, poderão apresentar diferenças significativas na riqueza de indivíduos. Embora os resultados obtidos demonstrem que existe uma influência marginalmente significativa na abundância da artropodofauna de solo ao longo do gradiente topográfico.

Mesmo com valores diferenciados de altura da serapilheira e número de árvores nos pontos amostrais, não foram verificadas diferenças significativas na riqueza $\left(r^{2}=0,185 ; p=0,283\right.$ / $\left.r^{2}=0,328 ; p=0,083\right)$ da artropodofauna de solo. Os resultados obtidos corroboram os de Zardo et al. (2010), onde o autor constatou que a serapilheira acumulada também não apresentou significativas influências na abundância e na riqueza das espécies por ela analisadas. Possivelmente, este resultado pode ter sido influenciado pelo escoamento da água da chuva e por processos erosivos. O escoamento pluvial superficial, bem como os processos erosivos em áreas de declínio, podem promover a movimentação e, consequentemente, a diminuição do acúmulo da serapilheira, dificultando o uso desta pelos artrópodes de solo.

\section{CONCLUSÃO}

Os resultados demonstraram que as ordens mais representativas nesta região de cerrado cerrado foram Hymenoptera, Araneae e Coleoptera. A ordem de menor representatividade foi a 
Scolopendromorpha. As morfoespécies de maior distribuição espacial foram Hymenoptera sp. 1, Hymenoptera sp. 2 e Coleoptera sp. 1. Foram observadas influências marginalmente significativas na abundância da artropodofauna de solo em relação ao gradiente topográfico, sendo que este resultado não se repete para a riqueza de espécies. Sugere-se a realização de estudo mais aprofundado e a longo prazo, que possa apresentar resultados mais relevantes, sanando assim dúvidas que possam surgir neste trabalho.

\section{AGRADECIMENTOS}

À Pró Reitoria de extensão e pesquisa acadêmica do UNIVAG Centro Universitário, pela infraestrutura física e acadêmica, à coordenação do Curso de Biologia na pessoa da Profa Ma. Márcia A. Rodrigues Nassarden, pelo carinho, apoio e inúmeras facilidades, ao Prof ${ }^{\circ}$ Me. Luiz Antônio Solino Carvalho, pelo valioso auxílio nas atividades desenvolvidas neste trabalho, à bióloga e técnica do laboratório de Zoologia, Paola Scame, pelo auxílio na triagem e identificação do Material biológico coletado.

\section{REFERÊNCIAS}

BATTIROLA, L. D. et al. Composição da comunidade de Formicidae (Insecta, Hymenoptera) em copas de Attaleaphalerata Mart. (Arecaceae), no Pantanal de Poconé, Mato Grosso, Brasil. Revista Brasileira de Entomologia, Curitiba, v. 49, p. 107-117, 2005.

CORREIA, M. E. F.; OLIVEIRA, L. C. M. Fauna de solo: aspectos gerais e metodológicos. Seropédica: Embrapa Agrobiologia, 2000. 46 p. Disponível em: <http://www.cnpab.embrapa.br/servicos/download/ doc112.pdf>. Acesso em: 03 jul. 2015.

COUTO, W. R. Biomonitoramento do solo e da água na avaliação de parâmetros ambientais da sub-bacia hidrográfica do baixo Apiaí-Guaçu. Dissertação (Mestrado em Tecnologia) Programa de Mestrado Profissional, Centro Estadual de Educação Tecnológica Paula Souza, São Paulo, 2008, 94 pag.

DIAS, S. C; SILVA, W. R; MARTINS, E. G; OSSES, F; BALDISSERA, R. Grupos funcionais de artrópodes de serapilheira de dois ambientes em uma área de extração de madeira na Amazônia Central. Curso de Ecologia da Floresta Amazônica, Manaus: INPA, 2004. Disponível em: http://www.inpa.gov.br/pdb_/download/efa/livro/2004/PDFs/pl1g6.df. Acessado em: 19 de julho de 2015.

FERREIRA, R. L.; MARQUES, M. M. G. S. M. A fauna de artrópodes de serapilheira de áreas de monocultura com Eucalyptus sp. e mata secundária heterogênea. Anais da Sociedade Entomológica Brasileira [online], v. 27, n. 3, p. 395-403, 1998.

GONZALEZ, M. I. M.; GALLARDO, J. F. El efecto hojarasca: una revisión. Anales de Edafologiay Agrobiologia, Madrid, v.41, n.5/6, p.1129-1157, 1982.

KNYSAK, I; MARTINS, R. Myriapoda. In: CA Joly; CE de Matos Bicudo. (Org.). Biodiversidade do Estado de São Paulo, Brasil: síntese do conhecimento ao final do século XX. São Paulo: Fapesp, v. 5, p. 65-72, 1999.

LOMOLINO, M. V. Elevation gradients of species -density: historical and prospective views. Global Ecology \& Biogeographya, Oklahoma, v. 10, p. 3-13, 2001.

LOPES, L. E. The range of the Curl-crested Jay: lessons for evaluating bird endemism in the South American Cerrado. Diversity and Distribuitions, v. 14, p. 561-568, 2008. 
LUIZÃO, R. C. C.; LUIZÃO, F. J.; PAIVA, R. Q.; MONTEIRO, T. F.; SOUZA, L. S.; KRUIJT, B. Variation of carbono and nitrogen cycling processes along a topographic gradient in a central Amazonian forest. Global Change Biology, Holanda, v. 10, p. 592-600, 2004.

MUDREK, R. J; MASSOLI, J. V.E. Estrutura da comunidade de artrópodes de solo em diferentes fitofisionomias da reserva particular do patrimônio natural - SESC Pantanal, Brasil. Holos, Natal, v. 1, p. 60-67, 2014.

NIMER, E. Climatologia no Brasil. Rio de Janeiro. Superintendência de Recursos Naturais e Meio Ambiente. IBGE Instituto Brasileiro de Geografia e estatística, 1979.

OLIVEIRA A. H. C. Padrões temporais de diversidade: dinâmica de assembleias de formigas de liteira (Hymenoptera: Formicidae) em $25 \mathbf{~ K m}^{2}$ de floresta Amazônica. 2013. p. 54. Dissertação de mestrado em Ciências Biológicas (entomologia). Instituto Nacional de Pesquisas da Amazônia (INPA). Manaus.

REYNAUD, P.A. Changes in understory avifauna along the Synnamaryriver (French Guyana, South America).Ornitologia Neotropical, Quebec, v 9, p. 51-70, 1998.

RICHARDS, B.N. Introduction to the soil ecosystem.NewYork.Longman Group Ltd., 1974, 266p.

ROCHA, G. O.; NETTO, M. C. B., LOZI, L. R. P. Diversidade, riqueza e abundância da entomofauna edáfica em área de cerrado do Brasil Central. Universidade Estadual de Goiás, Anápolis, GO. Disponível em: http://www.sebecologia.org.br/viiceb/resumos/1036a.pdf. Acessado dia 16 de agosto de 2015.

RUPPERT, E. E; FOX, R.S.; BARNES, R. D. Zoologia dos invertebrados. 7. ed. São Paulo: Roca, 2005, 1168p.

SANTOS, A. C. A.; SANTOS, J. M. L.; NECO. C. E. Riqueza, abundância e composição de artrópodes em diferentes estágios de sucessão secundária na caatinga. Biofar, Sergipe, v. 8, p. 151-159, 2012.

SILVA, P. A.; ROMIO, M. E. E.; VIEIRA, A. M.; CO RDEIRO, M. W. Estudo da diversidade de Arthropoda em fragmentos de mata no distrito de Rolim de Moura do Guaporé - RO. Revista Eletrônica da Facimed, Cacoal, v. 3, p. 315-321, 2011.

TOLEDO, J.J.; MAGNUSSON, W. E.; CASTILHO, C.V.; NASCIMENTO, H.E.M. How much variation in tree mortality is predicted by soil and topography in Central Amazonia? Forest Ecologyand Management, Nethelands, p.331-338, 2011.

TOKESHI, M. Species abundance patterns and community structure. Advances Ecological Research, New York, v. 24, p. 112-179, 1993.

WAGNER, D. e J.B. JONES. 2006. The impact of harvester ants on decomposition, of mineralization, litter quality, and availability of $\mathbf{N}$ to plants in the Mojave Desert. Soil Biol. Biochem. V. 38, p. 2593-2601.

WHITTAKER, R. H.; NIERNG, W. A.Vegetation of the Santa Catalina Mountains, Arizona, II.a gradient analysis of the South slope. Ecology, Durham, v. 46, p. 429-452, 1965.

ZARDO, C. D.; CARNEIRO, P. A.; LIMA, G. L.; FILHO, S. M. Comunidade de artrópodes associada a serrapilheira de cerrado e mata de galeria, na Estação Ecológica Serra das Araras- Mato Grosso, Brasil. Revista Uniara, Araraquara, v.13, p. 105-113, 2010. 